\title{
Effect of Pump Dithering at Each Stage of Cascaded Fiber Optical Parametric Amplifier
}

\author{
Fatin Nabilah Mohamad Salleh ${ }^{1}$, Nor Shahida Mohd Shah ${ }^{{ }^{2}}$, Nurulanati Othman ${ }^{3}$, \\ Rahmat Talib ${ }^{4}$, Munirah Ab. Rahman ${ }^{5}$ \\ ${ }^{1,3,4,5}$ Faculty of Electrical and Electronic Engineering, Universiti Tun Hussein Onn Malaysia, 86400 Batu \\ Pahat, Malaysia, Tel: 07-4537502/8304, Fax: 07-4538387 \\ ${ }^{2}$ Faculty of Engineering Technology, Universiti Tun Hussein Onn Malaysia, 86400 Batu Pahat, Malaysia, \\ Tel: 07-4537315/7595, Fax: 07-4536025 \\ ${ }^{3,4}$ Wireless and Radio Science Centre, Faculty of Electrical and Electronic Engineering, Universiti Tun \\ Hussein Onn Malaysia, 86400 Batu Pahat, Malaysia, Tel: 07-4537209, \\ Fax: 07-4536060 \\ *Corresponding author, e-mail: shahida@uthm.edu.my
}

\begin{abstract}
Cascaded fiber optical parametric amplifier (FOPA) can enhance gain and bandwidth. The gain and bandwidth can be further enhanced by dithering the FOPA pump. However, to our knowledge, the effects of a pump dithering at every stage of cascaded FOPA have not been discussed. The study of performance at every stage of cascaded FOPA is quite interesting and beneficial in designing the system. Here, we analyzed, using OptiSystem software, each stage of a cascaded FOPA, when there was a pump dithering and not. The results showed that the pump dithering enhanced the gain and broaden the bandwidth at every stage. The gain and bandwidth obtained with the pump dithering were $27 \mathrm{~dB}$ and 20 $\mathrm{nm}$, respectively. On the other hand, when there was no pump dithering, the gain and bandwidth were 9 $\mathrm{dB}$ and $12 \mathrm{~nm}$, respectively.
\end{abstract}

Keywords: fiber optical parametric amplifier; pump dithering; four-wave mixing

Copyright $\odot 2017$ Universitas Ahmad Dahlan. All rights reserved.

\section{Introduction}

Fiber optical parametric amplifiers (FOPAs) have obtained many research interest as the amplification bandwidth can be tuned to S-C-L band and surpass the bandwidth of erbiumdoped fiber amplifiers or Raman amplifiers [1]. Several researches were conducted on FOPAs to obtain high gain or broad bandwidth. One of the methods to enhance the gain or the bandwidth is by using cascaded FOPA [2-4].

Cascaded FOPA is FOPA with two or more active media, commonly, highly nonlinear fiber (HNLF). Devices such as isolators and fiber-Bragg gratings can be inserted in between the HNLFs to intensify the gain or the bandwidth of FOPA [5,6]. Besides that, dispersion compensating single mode fiber has been demonstrated to be in between the HNLFs which can act as a phase shifter [7]. The gain and bandwidth of cascaded FOPA can further be enhanced by dithering the pump of the FOPA [8].

In reference [8], the output at each stage of the cascaded FOPA was not discussed in details. Here, we extend the study of cascaded FOPA with and without pump dithering, by observing the signal power at each stage of the cascaded FOPA. The result at each stage is crucial to ensure that the cascaded FOPA is working smoothly.

In this work, the cascaded FOPA is simulated by using OptiSystem software. The cascaded FOPA has four stages of HNLF with different dispersion characteristics and lengths, as discussed in [8]. The gain and bandwidth obtained in the simulation are compared with the experimental results in [8] to see the validity of the simulation. The effects of pump dithering are compared with the cascaded FOPAs without the pump dithering. 


\section{Stimulated Brillouin Scathering (SBS) and Pump Dithering}

FOPA adopts the nonlinear effect of four-wave mixing, usually hindered by SBS that generate backward wave in HNLF, which limits the input power of FOPA. Accordingly, the SBS also implies a strict limit to the pump power that can be delivered to the HNLF. The SBS occurs when the power in optical fiber is more than SBS threshold. After the SBS power threshold, almost any additional power increase will be backscattered by SBS. The SBS power threshold is defined as:

$$
P_{t h} \approx \frac{21 k A_{e f f}}{g_{o} L_{e f f}}\left[\frac{\Delta v_{B} \Delta v_{P}}{\Delta v_{B}}\right],
$$

where $k$ is the wave polarization state, $A_{\text {eff }}$ is the effective area of the optical fiber, $L_{e f f}$ is effective interaction length, $g_{o}$ is the Brillouin gain parameter, $\Delta v_{\mathrm{B}}$ is Brillouin gain bandwidth, and $\Delta v_{\mathrm{P}}$ is incident pump linewidth.

From equation (1), it can be seen that the SBS power threshold depends on the pump linewidth. The SBS threshold can be increased by broadening the pump linewidth, or called as pump dithering. Pump dithering is a common method used to overcome the SBS in FOPA. By broadening the pump linewidth, the coherent buildup of the generally narrow SBS is reduced [9]. Pump dithering usually be done by phase modulated pump to more than the SBS bandwidth, which is typically tens of $\mathrm{MHz}$. The electrical signal driving the phase modulator is typically a few radio-frequency (RF) tones. Besides RF, a pseudo-random bit sequence or amplified spontaneous emission can also be used. This method can increase the SBS power threshold many times.

In addition, isolator can be used to reduce the back reflection light. Our setup combined both methods of SBS suppression: pump dithering and isolator.

\section{Structure of Cascaded FOPA}

The simulation setup can be referred from [8]. Figure 1 illustrates the structure of the cascaded FOPA with the pump dithering.

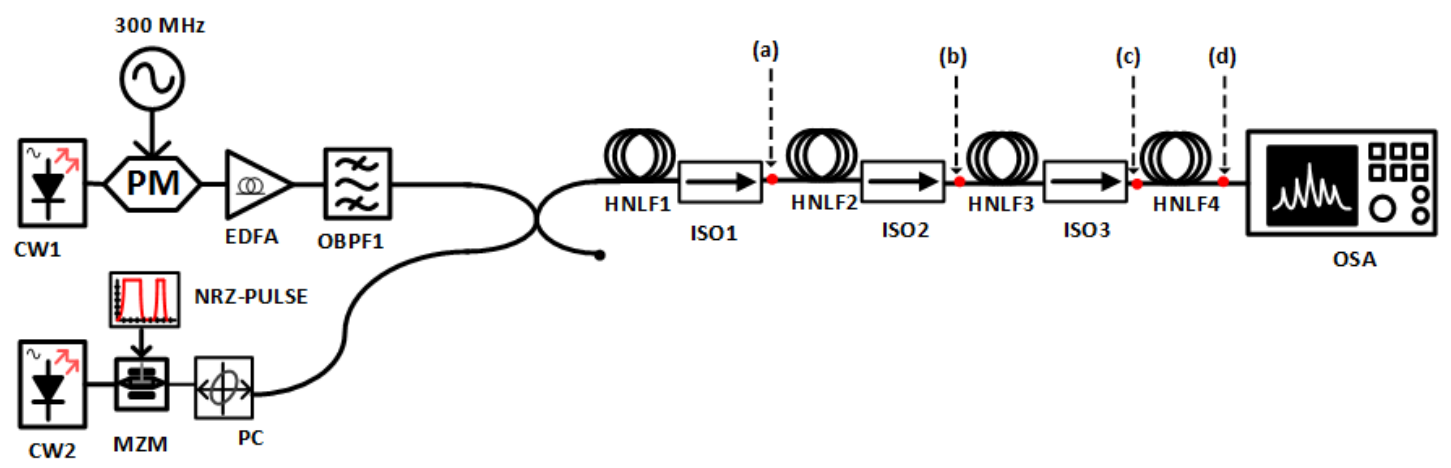

Figure 1. Simulation setup of cascaded FOPA with $300 \mathrm{MHz}$ RF tone

The pump light at $1554.1 \mathrm{~nm}$ is amplified by an erbium-doped fiber amplifier (EDFA). Then, it is filtered and coupled to a signal with a Wavelength Division Multiplexer (WDM) coupler. The signal is modulated by $10 \mathrm{Gbit} / \mathrm{s}$ data in on-off keying (OOK) format by using a Mach-Zender modulator. For the setup with pump dithering, RF tones are phase modulated in the pump at $300 \mathrm{MHz}$ to enhance the linewidth broadening and this has resulted in an extremely efficient SBS suppression. Subsequently, the signal is selected by a set of optical filter at the output. Table 1 lists the parameters of four HNLFs used at every stage of the FOPA. 
Table 1. The parameters of four HNLF

\begin{tabular}{ccccc}
\hline Parameter & HNLF1 & HNLF2 & HNLF3 & HNLF4 \\
\hline Length $(\mathrm{m})$ & 114 & 143 & 182 & 253 \\
Fiber attenuation $(\mathrm{dB} / \mathrm{km})$ & 0.86 & 0.86 & 0.77 & 0.77 \\
Zero dispersion wavelength $(\mathrm{nm})$ & 1541.6 & 1542.4 & 1535.8 & 1532.0 \\
Nonlinear coefficient $\left(\mathrm{W}^{-1} \mathrm{~km}^{-1}\right)$ & 11.7 & 11.7 & 11.7 & 11.7 \\
Effective area $\left(\mu \mathrm{m}^{2}\right)$ & 10.5 & 10.5 & 10.5 & 10.5 \\
\hline
\end{tabular}

\section{Result and Analysis}

For the result, first, we compare the gain and bandwidth obtained from our simulation with the gain and bandwidth obtained experimentally in [8] to validate our simulation. Figure 2 shows the FOPA gain, with and without pump dithering, obtained from the experimental and the simulation works. The black and gray curves with dots are the gain spectrum of experimental works taken from [8]. In contrast, the black and gray solid curves without dots are the simulation findings in this work. The black curve is the gain with a pump dithering while the gray curve is the gain without the pump dithering.

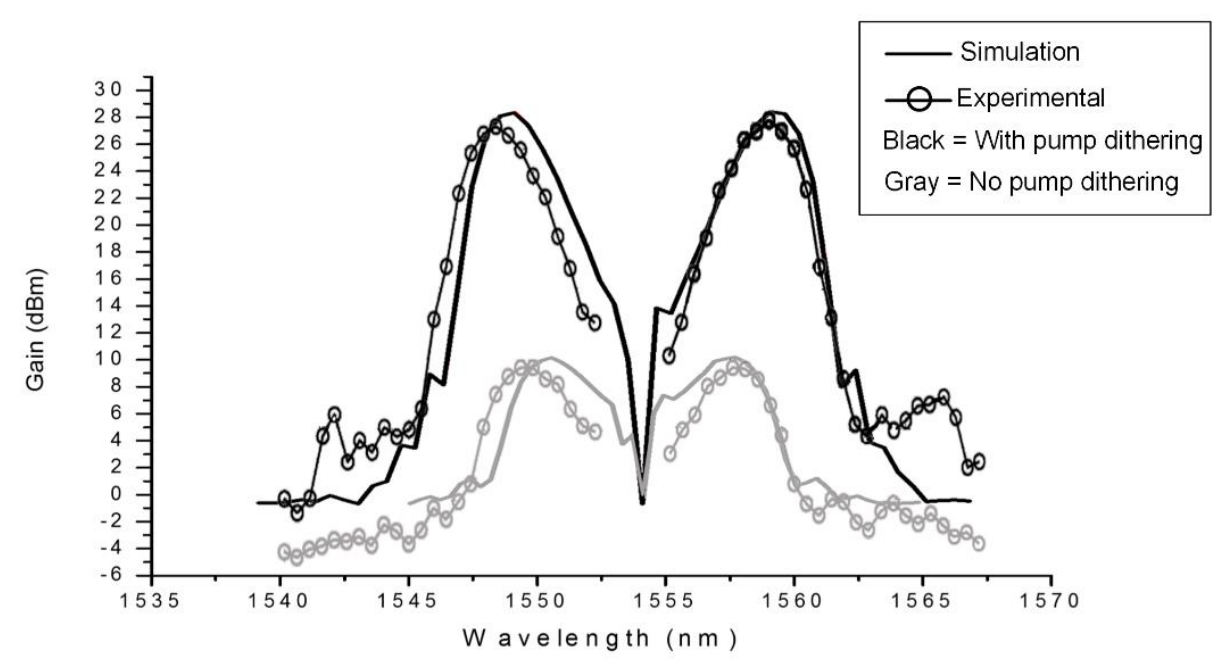

Figure 2. Gain for cascaded FOPA with pump dithering (black curve) and without pump dithering (gray curve). The solid curves are the results from simulation; while the curves with dots are the results taken from ref [8]

The simulation results obtained are almost similar to the experimental results taken from ref [8]. The simulated gain with and without pump dithering are $27 \mathrm{~dB}$ and $9 \mathrm{~dB}$, respectively. Meanwhile, the simulated bandwidth with and without pump dithering are $20 \mathrm{~nm}$ and $12 \mathrm{~nm}$, respectively. The gain and bandwidth for cascaded FOPA with a pump dithering are higher than the cascaded FOPA without pump dithering. The pump dithering has suppressed the SBS by dithering the pump light. The pump linewidth is broadened and increase the SBS threshold. The maximum power of the pump is delivered to the HNLF. The gain for both cases shows a good agreement and the investigation at the output power at each stage is conducted. The investigation is continued by observed the signal power at each stage. The signal power at each stage is crucial as the gain is observed the signal power at the input and output. Figure 3 shows the signal power at each stage of cascaded FOPA without a pump dithering. 

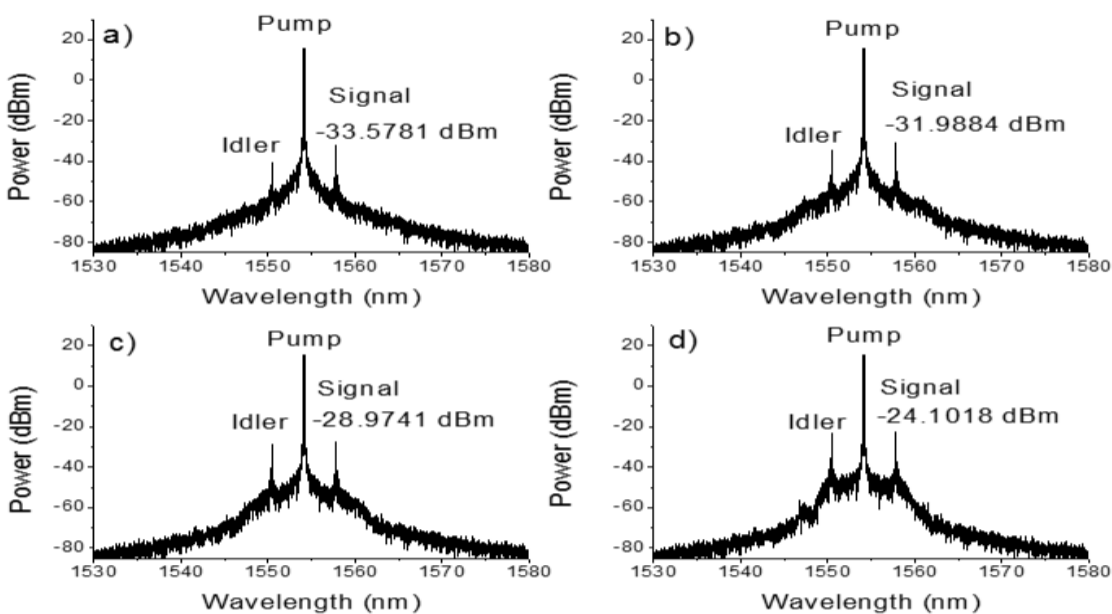

Figure 3. The output of every stage (no pump dithering)

As can be seen in Figure 3, the signal wavelength, $\lambda_{s}$ is $1557.8 \mathrm{~nm}$. Meanwhile the first idler wavelength, $\lambda_{1}$ is at $1550.4 \mathrm{~nm}$. The signal power at the first stage is $-33.5781 \mathrm{dBm}$ as shown in Figure 3(a). The signal power at the second stage of cascaded FOPA without the pump dithering is $-31.9884 \mathrm{dBm}$ as shown in Figure $3(\mathrm{~b})$. Subsequently, the power is amplified with a signal power achieved $-28.9741 \mathrm{dBm}$ as shown in Figure 3(c). At the last stage, the signal power is $-24.1018 \mathrm{dBm}$ as shown in Figure $3(\mathrm{~d})$. Then, the cascaded FOPA with a pump dithering is investigated by placing a $300 \mathrm{MHz} R \mathrm{~F}$-tone at the pump. Figure 4 shows the output at each stage for cascaded FOPA with a $300 \mathrm{MHz}$ RF-tone.
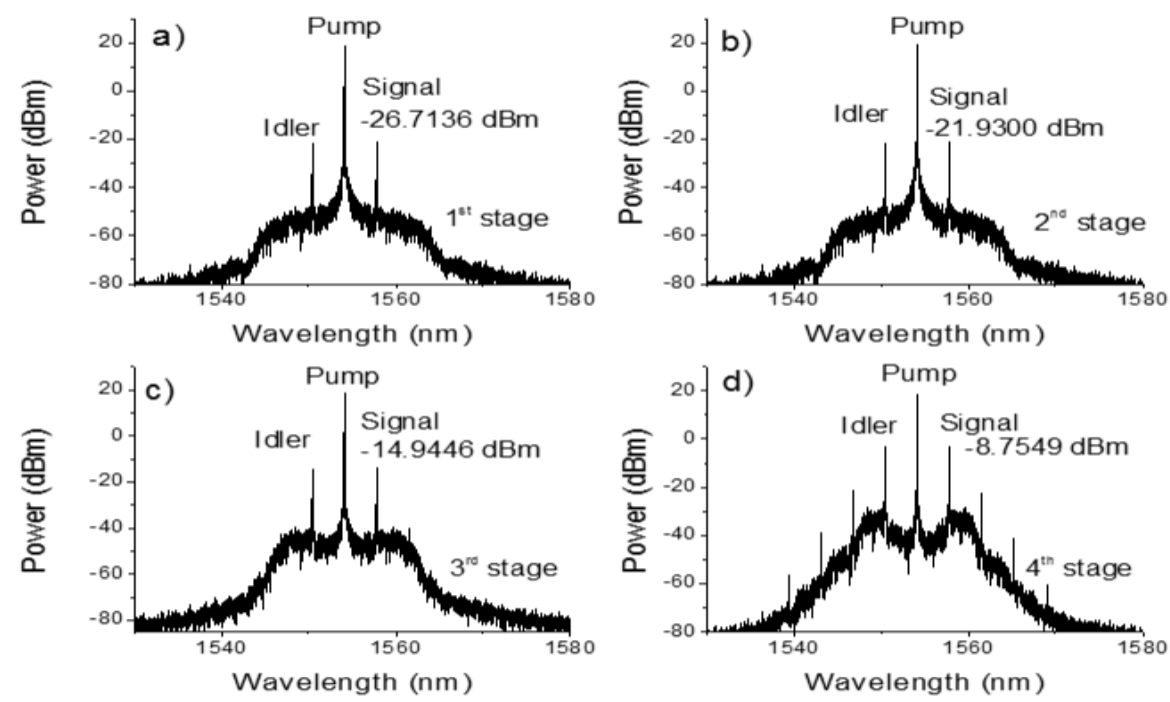

Figure 4. The output at every stage of cascaded FOPA (with pump dithering)

Figure 4 shows the output at each stage of the cascaded FOPA with a pump dithering. At the first stage, the signal power reached $-26.7136 \mathrm{dBm}$ as shown in Figure 4(a). Figure 4(b) indicates the result at the second stage where the idler power increases to $-21.9300 \mathrm{dBm}$. The increasing signal power is also observed at the next stages as the third stage and the fourth stage signal power are -14.9446 and $-8.7549 \mathrm{dBm}$ as can be seen in Figure 4(c) and Figure 
4(d). The signal power of the cascaded FOPA with a pump dithering at every stage is higher than the signal power of the cascaded FOPA without a pump dithering. This shows a good agreement with the gain spectrum as the power is amplified inside the cascaded FOPA. Table 2 states the signal powers at every stage for both cases.

Table 2. The signal powers at every stage for cascaded FOPA with and without pump dithering

\begin{tabular}{ccc}
\hline Stage & No pump dithering $(\mathrm{dBm})$ & With pump dithering $(\mathrm{dBm})$ \\
\hline $1^{\text {st }}$ stage & -33.5781 & -26.7316 \\
$2^{\text {nd }}$ stage & -31.9884 & -21.9300 \\
$3^{\text {rd }}$ stage & -28.9741 & -14.9446 \\
$4^{\text {th }}$ stage & -24.1018 & -8.7549 \\
\hline
\end{tabular}

The pump and idler powers are also examined. Table 3 shows the pump power and idler powers for the both cases. The pump decreases at every stage for both cases. This is due to the optical power transfer from the pump to the signal and idler. The pump power at each stage for cascaded FOPA with a pump dithering is higher than the cascaded FOPA without a pump dithering. The idler power is also increases at every stage for both cases due to power transfer from the pump. The effect of pump dithering in cascaded FOPA has been compared and it clearly shows that pump dithering also affects the pump and idler power.

Table 3. The pump and idler powers at each stage for both cases

\begin{tabular}{ccrcr}
\hline Stage & \multicolumn{2}{c}{ No pump dithering $(\mathrm{dBm})$} & \multicolumn{2}{c}{ With pump dithering $(\mathrm{dBm})$} \\
\cline { 2 - 5 } & Pump power & Idler power & Pump power & Idler power \\
\hline $1^{\text {st }}$ stage & 15.9437 & -39.1205 & 19.2852 & -28.9943 \\
$2^{\text {nd }}$ stage & 15.8205 & -32.8605 & 19.1614 & -21.7156 \\
$3^{\text {rd }}$ stage & 15.6798 & -27.5765 & 19.0162 & -14.0660 \\
$4^{\text {th }}$ stage & 15.4827 & -21.7035 & 18.7543 & -3.8718 \\
\hline
\end{tabular}

\section{Conclusion}

In conclusion, the pump dithering improves the gain and bandwidth of the cascaded FOPA. The gain is enhanced from $9 \mathrm{~dB}$ to $27 \mathrm{~dB}$ while the bandwidth is broadened from $12 \mathrm{~nm}$ to $20 \mathrm{~nm}$. The pump dithering suppresses the SBS by increasing the pump linewidth. The characteristics at each stage also shown an improvement where the signal power at each stage is growing. The signal power at every stage is low when there is no pump dithering. At the final stage of cascaded FOPA, the signal power is $-8.7549 \mathrm{dBm}$ with a pump dithering and -24.1018 $\mathrm{dBm}$ without a pump dithering.

\section{Acknowledgement}

This work is supported by Graduate Incentive Scheme (GPPS) vot U454 granted by Universiti Tun Hussein Onn Malaysia and Fundamental Research Grant Scheme (FRGS) vot 1537 granted by Ministry of Higher Education Malaysia.

\section{References}

[1] Othman N, Mohd Shah, NS, Tay KG, Cholan NA. The influence of fiber parameters to the fiber optical parametric amplifier gain spectrum. International Conference on Advances in Electrical, Electronic and Systems Engineering. 2016; 326-330.

[2] Nguyen TH, Inoue T, Tanizawa K, Petit S, Oikawa Y, Takasaka S, Yagi T, Namiki S. Counterdithering pump scheme for cascaded degenerate FWM based wavelength converter. Conference on Optical Fiber Communication, Technical Digest Series. 2014; 31--33.

[3] Takasaka S, Mimura Y, Matsuura H, Morimoto M, Takahashi M, Sugizaki R. FOPA with Flat 21-dB Gain and NF less than 4-dB using Alternately Concatenated Pump-Phase Shifters and HNLFs. Optical Fiber Communication Conference/National Fiber Optic Engineers Conference. 2013; 1-3.

[4] Ruihong X, Yu C, Sang X, Hsu D. Research on QPM and Its Cascaded Structure Applied in FOPA. Cybern. Inf. Technol. 2016; 16: 50-58. 
[5] Wong KKY, Shimizu K, Uesaka K, Kalogerakis G, Marhic ME, Kazovsky LG. Continuous-Wave Fiber Optical Parametric Amplifier with 60-dB Gain Using a Novel Two-Segment Design. IEEE Photonics Technol. Lett. 2003; 15: 1707-1709.

[6] Zhu H, Luo B, Pan W, Yan L, Xiang S, Wen K. Gain enhancement of fiber optical parametric amplifier via introducing phase-shifted fiber Bragg grating for phase matching. J Opt Soc Am B. 2012; 29 : 1497.

[7] Xia M, Wu Y, Xia S, Liu G, Yang H, Ping Z, Wang T. Power Equalization for Non-degenerate Cascaded Phase-sensitive Fiber-optic Parametric Amplifier. 4th International Conference on Computer Science and Network Technology. 2015; 1236-1239.

[8] Lundström C, Malik R, Grüner-Nielsen L, Corcoran B, Olsson SLI, Karlsson M, Andrekson PA. Fiber optic parametric amplifier with 10-dB net gain without pump dithering. IEEE Photonics Technol. Lett. 2013; 25: 234--237.

[9] Coles JB, Kuo BPP, Alic N, Moro S, Bres CS, Chavez Boggio JM, Andrekson PA, Karlsson M, Radic $\mathrm{S}$. Bandwidth-efficient phase modulation techniques for stimulated Brillouin scattering suppression in fiber optic parametric amplifiers. Opt. Express. 2010; 18: 18138--18150. 